\title{
Efficient Algorithms for Prefix and General Prefix Computations on Distributed Shared Memory Systems with Applications *
}

\author{
V. Kamakoti \\ N. Balakrishnan \\ Supercomputer Education and Research Centre \\ Indian Institute of Science, Bangalore - 560 012, India \\ \{kama,balki\}@serc.iisc.ernet.in
}

\begin{abstract}
The paper presents efficient scalable algorithms for performing Prefix (PC) and General Prefix $(G P C)$ Computations on a Distributed Shared Memory $(D S M)$ system with applications.
\end{abstract}

\section{Introduction}

Prefix $(P C)$ and General Prefix (GPC) Computations are generic techniques that can be used to design sequential and parallel algorithms for a number of problems from diverse areas $[1,5,6]$. In $[6]$ an $O(\log n)$ time, $O(n)$ processor, $C R E W P R A M$ (shared memory) parallel algorithm for the $G P C$ is presented. This algorithm implies an $O(n \log n)$ time sequential algorithm.

Based on memory organization, parallel computing systems fall into two categories: Shared Memory systems and Distributed Memory systems. Shared Memory systems are relatively easy to program (due to a single address space) but less scalable than distributed memory systems. A software abstraction in which a distributed memory system can be viewed as a system with a single address space results in a system that is both scalable and easy to program. Such systems are called Scalable Shared Memory Systems or Distributed Shared Memory Systems. The BDM model of computing, from a users perspective, offers the advantage of ease of programming of shared memory systems while from a systems perspective, provides the advantage of scalability akin to message passing systems.

$P C$ and $G P C$ are generic techniques for algorithm design. Therefore, a solution for them implies a solution for a variety of problems in diverse areas that include Computational Geometry, Graph Theory, Sorting etc. Some of the routines are recursive and were successfully implemented on the SP2 which is a Distributed Memory Machine. This gives some pointers for automatically parallelizing recursive programs. The paper also discusses the suitability of the $B D M$ model [3] for the IBM's SP2. This implies that the

\footnotetext{
*This work was supported in part by the IISC-TISL/IBM joint project (No. TISL/SERC/NB/02) on High Performance Computing Using Distributed Shared Memory under the Shared University Research Program
}

\begin{tabular}{|l|cccccc|}
\hline$m$ & 1 & 2 & 3 & 4 & 5 & 6 \\
\hline$f$ & 1.2 & 1.6 & 1.3 & 1.2 & 1.7 & 1.4 \\
$C_{m}$ & 1.2 & 1.6 & 1.6 & 1.6 & 1.7 & 1.7 \\
$Y$ & 1 & 4 & 2 & 3 & 4.5 & 3.5 \\
$D_{m}$ & $\phi$ & 1.2 & 1.2 & 1.3 & 1.6 & 1.3 \\
\hline
\end{tabular}

$n=6, *=\max , \rho=<, \phi=$ not defined.

Figure 1: Example for Prefix Maxima and General Prefix Maxima

model can serve as a tool for the programmer to design and analyse the algorithm, by deriving analytical expressions for the time/space complexity before actually implementing the same. In this regard, our paper can also serve as a case study for the users. We now formally define $P C$ and $G P C$ with an example shown in Figure 1.

\section{Definition 1 (Prefix Computation $(P C)$ )}

Let $(f(1), f(2), \cdots, f(n))$ be a given sequence of elements. The problem is to compute the sequence of prefixes $C_{m}=f(1) * f(2) * \cdots * f(m), 1 \leq m \leq n$, where, * is an arbitrary binary associative operator defined on the $f$-elements.

Definition 2 (GPC [6]) Let $(f(1), f(2), \cdots, f(n))$ and $(y(1), y(2), \cdots, y(n))$ be two given sequences of elements. Let $Y=\{y(1), \ldots, y(m)\}$. Let $\rho$ be a binary relation such that for $y_{i}, y_{j} \in Y, i \neq j$, either $\left(y_{i} \rho y_{j}\right)$ or $\left(y_{j} \rho y_{i}\right)$. The problem is to compute the sequence of general prefixes $D_{m}=f\left(j_{1}\right) * f\left(j_{2}\right) * \cdots * f\left(j_{k}\right), 1 \leq$ $m \leq n$, where, $*$ is an arbitrary binary associative operator defined on the $f$-elements and $j_{i}$ 's are indices such that

(a). $j_{i}<j_{i+1}, 1 \leq i<k$;

(b). $j_{i}<m, 1 \leq i \leq k$; and,

(c). $y\left(j_{i}\right) \rho y(\bar{m}), \overline{1} \leq i \leq k$.

It is easy to see that $P C$ is a special instance of $G P C$. An efficient algorithm for $P C$ is reported in [4]. 


\section{$1.1 \quad$ Preliminaries}

The $B D M$ model [3] is defined in terms of four parameters: number $p$ of processors, maximum initial latency time $\tau$ taken for a processor to receive the packet it requested from some other processor, time $\sigma$ taken to inject a word into or receive a word from the network, and number $m$ of consecutive words sent during each transfer. The processors are connected to a common communication network. Data are communicated between processors via point-to-point messages in blocks of $m$ consecutive words rather than a single word. This is done keeping in mind the spatial locality of programs in execution. Let $P R_{i}, 0 \leq i \leq p-1$ denote the $i^{\text {th }}$ processor in the $B D M$ system. Any processor can communicate with any other processor, but the time for communication depends upon the latency and bandwidth of the network, as described in the following facts about the $B D M$ given in [3]:

1. No processor can send or receive more than one packet (a block of $m$ consecutive words) at a time.

2. The model allows the initial placement of input data in the local memories of the processors and the memory latency hiding technique of pipelined prefetching.

3. If $\pi$ is any permutation on $p$ elements, then, a remote memory request for $b$ words issued by every processor $P R_{i}$ and destined for processor $P R_{\pi(i)}$ can be completed in $\tau+m \sigma\left\lceil\frac{b}{m}\right\rceil$ time for all processors $P R_{i}, 0 \leq i \leq p-1$, simultaneously. $k$ remote access requests issued by $k$ distinct processors and destined to the same processor will require $k(\tau+m \sigma)$ time to be completed, and the requests will be served in arbitrary order. $k$ prefetch read operations issued by a processor can be completed in $\tau+k$ in $\sigma$ time, using pipelined prefetching. $k$ prefetch read operations of $k$ blocks of $\left\lceil\frac{n}{p}\right\rceil$ words each, can be completed in $\tau+\sigma k m\left\lceil\frac{n}{p m}\right\rceil$ time.

4. There are two time-complexity measures for a parallel algorithm on the $B D M$ model; the computation time $T_{\text {comp }}$, and the communication time $T_{\text {comm }}$. The measure $T_{\text {comp }}$ refers to the maximum of the local computations performed on any processor as measured in the model of computation supported by it. The measure $T_{\text {comm }}$ refers to the total amount of communication time spent by the overall algorithm in accessing remote data.

Henceforth, without loss of generality we assume that $n$ is a multiple of $p$ and $p$ is a power of two.

Definition 3 ((IED) storage) $A$ sequence $F=$ $(f(1), f(2), \ldots, f(n))$ of $n$ elements is said to be Inorder Equally Distributed IED stored on a two-dimensional array $B\left[1 . . \frac{n}{t}: 0 . . t-1\right]$ in some $t \leq p$ processors $P R_{j_{0}}, P R_{j_{1}}, \ldots, P R_{j_{t-1}}$ of a $B D M$ machine if and only if,
$B[j, i]=f(i *(n / t)+j)$, for $0 \leq i \leq t-1,1 \leq$ $j \leq n / t$

$\left(B[1, i], B[2, i], \ldots, B\left[\frac{n}{t}, i\right]\right)$ are stored in processor $P R_{j_{i}}$ in this order, $0 \leq i \leq t-1$.

Before presenting the algorithm we define some functions that are used by the algorithm.

1. RANDOMROUTE: A randomized function which routes the data stored in each of the processors to their respective destinations. The input to this function is a $\frac{n}{p} \times p$ array $A$ of $n$ elements initially stored one column per processor in a $p$ processor $B D M$ machine. Each element of $A$ consists of a pair $($ data, $i)$, where $i$ is the index of the processor to which the data has to be relocated. For details refer Theorem A.1 in Appendix A. .

2. BDPRECOMP: Performs prefix computation on a sequence $I E D$ stored on a $p$-processor $B D M$ machine. For details refer Theorem A.2 in Appendix $A$.

\section{Merging Sorted Lists on a $B D M$ ma- chine}

We will first present an overview of the function which merges two given sorted lists on a $B D M$ machine and then, present its implementation. The function is a modification of Batcher's odd-even merge [2]. Without loss of generality we assume $p=2^{b}$, for some integer $b>0$. The two given sorted lists $L_{1}$ and $L_{2}$ of $\frac{n}{2}$ elements each are such that $L_{1}$ is $I E D$ stored in order in the processors $\left(P R_{0}, P R_{1}, \ldots, P R_{\frac{p}{2}-1}\right)$ and $L_{2}$ is $I E D$ stored in order in the processors $\left(P R_{\frac{p}{2}}, P R_{\frac{P}{2}+1}, \ldots, P R_{p-1}\right)$, $\left\lceil\frac{n}{p}\right\rceil$ elements per processor. We call the data stored within a single processor as a block. As in odd-even merge we do the following steps 1 and 2 in parallel:

1. Recursively merge the blocks stored in processors occupying even numbered positions/subscripts. In other words, the sorted sublist of $L_{1}$ stored in $\left(P R_{0}, P R_{2}, \ldots, P R_{\frac{p}{2}-2}\right)$ and the sorted sublist of $L_{2}$ stored in $\left(P R_{\frac{p}{2}}, P R_{\frac{p}{2}+2}, \ldots, P R_{p \sim 2}\right)$ are merged and the merged list is stored in $\left(P R_{0}, P R_{2}, \ldots, P R_{\frac{p}{2}-2}, P R_{\frac{p}{2}}, P R_{\frac{p}{2}+2}, \ldots, P R_{p-2}\right)$.

2. Recursively merge the blocks stored in processors occupying odd numbered positions/subscripts. In other words, the sorted sublist of $L_{1}$ stored in $\left(P R_{1}, P R_{3}, \ldots, P R_{P_{-1}}\right)$ and the sorted sublist of $L_{2}$ stored in $\left(P R_{\frac{p}{2}+1}, P R_{\frac{p}{2}+3}, \ldots, P R_{p-1}\right)$ are merged and the merged list is stored in $\left(P R_{1}, P R_{3}, \ldots, P R_{\frac{p}{2}-1}, P R_{\frac{p}{2}+1}, P R_{\frac{p}{2}+3}, \ldots, P R_{p-1}\right)$.

3. For all $k, 0 \leq k \leq p-1$, do in parallel if $k$ is odd and $k \neq p-1$, then, merge the block stored in $P R_{k}$ with the block stored in $P R_{k+1}$. 
4. The final sorted list is stored in order in $\left(P R_{0}, P R_{1}, \ldots, P R_{p-1}\right)$.

The procedure is similar to the odd-even merge [2] except that we treat every element of the latter as a block of sorted elements. The following function BlockMerge gives the recursive DSM implementation of the above method.

Function BlockMerge $\left(B L_{1}, B L_{2}, i, t, a\right):(B L)$ Input: Two sorted lists $L_{1}$ and $L_{2}$ each of length $t(n / p)$ elements such that $L_{1}$ is $I E D$ stored on an array $B L_{1}$ in the processors $P R_{i}, P R_{i+a}, \ldots, P R_{i+(t-1) a}$ and $L_{2}$ is $I E D$ stored on an array $B L_{2}$ in the processors $P R_{i+t a}, P R_{i+(t+1) a}, \ldots, P R_{i+(2 t-1) a}$.

Output: The sorted list $L=\operatorname{Merge}\left(L_{1}, L_{2}\right)$, of length $2 t(n / p) I E D$ stored on an array $B L$ in the processors $P R_{i}, P R_{i+a}, \ldots, P R_{i+(2 t-1) a}$

Begin /* W.l.g we assume that $t$ is a power of two */

1. If $(t=1)$ then /* only two blocks to be merged $* /$ $P R_{i}$ and $P R_{i+a}$ hold the sorted lists $B L_{1}$ and $B L_{2}$ of $\left\lceil\frac{n}{p}\right\rceil$ elements each respectively. $P R_{i}$ reads the list $B L_{2}$ stored in $P R_{i+a}$ and sequentially merges it with $B L_{1}$ and stores one half of the merged list in itself, in the array $B L(j, i), 1 \leq j \leq n / p$ and the other half in $P R_{i+a}$ in the array $B L(j, i+a), 1 \leq j \leq n / p$. return $(B L)$; exit

Do Steps 2 and 3 in Parallel $/ * t>1 * /$

2. $B L=B$ lockMerge $\left(B L_{1}, B L_{2}, i, \frac{t}{2}, 2 a\right)$

/* In step 2 the part of the lists $B L_{1}$ and $B L_{2}$ stored in processors, $P R_{i+k a}$, for even $k \geq 0$ in the sequence $\left(P R_{i}, P R_{i+a}, \cdots, P R_{i+(2 t-1) a}\right)$ are merged $*$ /

3. $B L=B$ lockMerge $\left(B L_{1}, B L_{2}, i+a, \frac{t}{2}, 2 a\right)$

$/ *$ In step 3 the part of the lists $B L_{1}$ and $B L_{2}$ stored in processors, $P R_{i+k a}$, for odd $k \geq 1$ in the sequence $\left(P R_{i}, P R_{i+a}, \cdots, P R_{i+(2 t-1) a}\right)$ are merged $* /$

4. For all Processors $P R_{i+k a}$, such that $1 \leq k \leq t-3$ and $k$ is an odd number do in parallel

$P R_{i+k a}$ reads the sorted list $B L(j, i+(k+1) a)$,

$1 \leq j \leq n / p$ from $P R_{i+(k+1) a}$ and merges sequentially the same with the list $B L(j, i+k a), 1 \leq j \leq n / p$ stored in it. Then, it stores one half of the merged list in itself, in the array $B L(j, i+k a), 1 \leq j \leq n / p$ and the other half in $P R_{i+(k+1) a}$ in the array $B L(j, i+(k+1) a), 1 \leq j \leq n / p$.

/*In step 4 all the processors, $P R_{i+k a}$, for odd $k \geq 1$, in the sequence $\left(P R_{i}, P R_{i+a}, \cdots, P R_{i+(2 t-1) a}\right)$ except the last processor $P R_{i+(2 t-1) a}$, merges the $B L$ array stored in it with the $B L$ array of the immediately succeeding processor $P R_{i+(k+1) a}$.*/

5. $\operatorname{return}(B L)$

End.

From the assumptions of the $B D M$ model we see that step 4 of the above function takes $\tau+\sigma m\left\lceil\frac{n}{p m}\right\rceil$ communication time. Step 4 takes $O\left(\frac{n}{p}\right)$ computation time. Hence for the whole procedure,
$T_{\text {comm }}(t)=T_{\text {comm }}\left(\frac{t}{2}\right)+\tau+\sigma m\left\lceil\frac{n}{p m}\right\rceil$,

$T_{\text {comm }}(1)=\tau+\sigma m\left\lceil\frac{n}{p m}\right\rceil$ (from Step 1).

$T_{\text {comp }}(t)=T_{\text {comp }}\left(\frac{t}{2}\right)+O\left(\left\lceil\frac{n}{p}\right\rceil\right)$,

$T_{\text {comp }}(1)=O\left(\left\lceil\frac{n}{p}\right\rceil\right)$ (from Step 1$)$.

Theorem 1 Function BlockMerge $\left(B L_{1}, B L_{2}, i, t, a\right)$

takes $O\left(\frac{n \log _{2} t}{p}\right)$ computation time and $O\left(\left(\tau+\sigma m\left\lceil\frac{n}{p m}\right\rceil\right) \log _{2} t\right)$ communication time.

Corollary 1 Two lists each of size $n$ can be merged using $p$ processors on a distributed memory system in $O\left(\frac{n \log _{2} p}{p}\right)$ computation time and $O\left(\left(\tau+\sigma m\left\lceil\frac{n}{p m}\right\rceil\right) \log _{2} p\right)$ communication time.

\section{The Ranking Problem}

We will now define a subroutine $R A N K P$ to solve the ranking problem. $R A N K P$ will be used by the main algorithm. First we define the following notations. Let $L=\left(l_{1}, l_{2}, \ldots, l_{t(n / p)}\right)$ and $L D=\left(\left(y_{1}, d_{1}\right),\left(y_{2}, d_{2}\right), \ldots,\left(y_{t(n / p)}, d_{t(n / p)}\right)\right)$ be two sequences IED stored on the arrays $B L$ and $B L D$ respectively in the processors $P R_{c}, P R_{c+1}, \ldots, P R_{c+(t-1)},((n / p)$ elements per processor). Similarly, let $R=\left(r_{1}, r_{2}, \ldots, r_{t(n / p)}\right)$ and $R D=\left(\left(y_{1}^{\prime}, d_{1}^{\prime}\right),\left(y_{2}^{\prime}, d_{2}^{\prime}\right), \ldots,\left(y_{t(n / p)}^{\prime}, d_{t(n / p)}^{\prime}\right)\right)$ be two sequences $I E D$ stored on the arrays $B R$ and $B R D$ respectively in the processors $P R_{c+t}, P R_{c+t+1}, \ldots, P R_{c+(2 t-1)}$. From Definition 3 we see that, $B L D[j, i]=\left(y_{i *(n / p)+j}, d_{i *(n / p)+j}\right), 1 \leq$ $j \leq n / p, 0 \leq i \leq t-1$, and is stored in the processor $P R_{c+i}$. Let $d_{i *(n / p)+j}=(j, c+i$,dat $)$, where dat denotes some data of constant size. Similarly $B R D[j, i]=\left(y_{i *(n / p)+j}^{\prime}, d_{i *(n / p)+j}^{\prime}\right), 1 \leq j \leq n / p$, $0 \leq i \leq t-1$, and is stored in the processor $P \widetilde{R_{c+t+i}}$. Let $d_{i *(n / p)+j}^{\prime}=(j, c+t+i, d a t)$, where dat denotes some data of constant size. Let $S L$ be the sorted sequence of $L D$, sorted on the $y$ values. $S L$ is $I E D$ stored on the arrays $B S L$ in the processors $P R_{c}, P R_{c+1}, \ldots, P R_{c+(t-1)}$. Let $S R$ be the sorted sequence of $R D$, sorted on the $y^{\prime}$ values. $S R$ is $I E D$ stored on the arrays $B S R$ in the processors $P R_{c+t}, P R_{c+t+1}, \ldots, P R_{c+(2 t-1)}$.

Definition 4 (Ranking Problem) The problem is to merge the two sorted lists $S L$ and $S R$ (on the $y$ and $y^{\prime}$ values) into a single sorted list $P L$ and do the following:

1. for every element $P L(j), 1 \leq j \leq 2 t(n / p)$, if $P L(j)\left(=\left(y_{i}, d_{i}\right)\right) \in L D$, then find the two consecutive elements $\left(y_{j}^{\prime}, d_{j}^{\prime}\right)$ and $\left(y_{k}^{\prime}, d_{k}^{\prime}\right)$ in $S R$ such that $y_{i}$ lies inbetween $y_{j}^{\prime}$ and $y_{k}^{\prime}$. Let $f_{i}=\left(d_{j}^{\prime}, d_{k}^{\prime}\right)$; and,

2. for every element $P L(j), 1 \leq j \leq 2 t(n / p)$, if $P L(j)\left(=\left(y_{i}^{\prime}, d_{i}^{\prime}\right)\right) \in R D$, then find the two consecutive elements $\left(y_{j}, d_{j}\right)$ and $\left(y_{k}, d_{k}\right)$ in $S L$ such that $y_{i}^{\prime}$ lies inbetween $y_{j}$ and $y_{k}$. Let $f_{i}=\left(d_{j}, d_{k}\right)$. 
Let $F=\left(f_{1}, f_{2}, \ldots, f_{2 t(n / p)}\right)$ and $P L$ be $I E D$ stored on the arrays $T F$ and $R P L$ in the processors $P R_{c}, P R_{c+1}, \ldots, P R_{c+(2 t-1)}$.

Function $R A N K P(B S L, B S R, c, t):(R P L, T F)$ Begin

1. $R P L=B$ lockM erge $(B S L, B L R, c, t, 1)$ (merging on the $y$ and $y^{\prime}$ values).

2. For each processor $P R_{c+i}, 0 \leq i \leq 2 t-1$ do in parallel

For $1 \leq j \leq n / p$

- Let $R P L[j, i]=(u, v)$. From entry $v$ we can find out whether the element belongs to $B S L$ or $B S R$.

- If the element $R P L[j, i]\left(=\left(y_{k}, d_{k}\right)\right)$ belongs to $B S L$ then, set $T E M P[j, i]=\left(i *(n / p)+j, d_{k}\right)$, else $T E M P[j, i]=(0,0)$

3. Do a prefix maxima on the TEMP array using the function $B D P R E C O M P$ (refer Theorem A.2 of Appendix A). Let the routine return the array $T E M P^{\prime}$.

4. For each processor $P R_{\mathrm{c}+i}, 0 \leq i \leq 2 t-1$ do in parallel

For $1 \leq j \leq n / p^{p}$

- Let $R P L[j, i]=(u, v)$. From entry $v$ we can find out whether the element belongs to $B S L$ or $B S R$. If the element $R P L[j, i]\left(=\left(y_{k}^{\prime}, d_{k}^{\prime}\right)\right)$ belongs to $B S R$ then, let $T E M P^{\prime}[j, i]=\left(u^{\prime}, v^{\prime}\right)$. It is easy fo see that $v^{\prime}$ is the first entry of the tuple $f_{k}^{\prime}$.

/* In a similar fashion we can calculate the second entry of the tuple $f_{k}^{\prime}$ for the elements in $B S R$. We can also calculate for all elements $R P L[j, i]$ belonging to $B S L$, the corresponding $f$-tuple in a similar fashion. Hence we can compute the $T F$ array.*/

End.

Note that steps 2 and 4 take $O(n / p)$ computation time. Theorems 1 and A.2 imply the following theorem.

Theorem 2 Function $R A N K P(B S L, B S R, c, t)$ takes $O\left(\frac{n \log _{2} t}{p}+\frac{\tau \log _{2} t}{\sigma m \log _{2}\left(\frac{\tau}{\sigma m}+1\right)}\right)$ computation time and $O\left(\left(\tau+\sigma m\left\lceil\frac{n}{p m}\right\rceil\right) \log _{2} t\right)$ communication time.

\section{Algorithm for the $G P C$ on the $B D M$ model}

The overview of the algorithm is as follows. The algorithm is a recursive one, which basically boils down to each processor $P R_{i}, 0 \leq i \leq p-1$, performing the $G P C$ for the elements stored within itself sequentially, using the algorithm presented in [6] and then, merging the $p$ individual solutions to get the final solution.

\subsection{Sequential Algorithm for the GPC problem}

In this section we will briefly describe the algorithm for the $G P C$, presented in [6]. It is a Divide and Conquer recursive algorithm. It takes as an input two sequences $S F=(s f(1), s f(2), \ldots, s f(k))$ and $S Y=$ $(s y(1), s y(2), \ldots, s y(k))$ and returns as an output, four sequences $O S Y, S D, S P, S E$. Note that, $S F$ corresponds to the $f$ sequence and $S Y$ corresponds to the $y$ sequence of Definition 2. Before defining these arrays we introduce the following notion.

Definition 5 Given the input sequences $S F$ and $S Y$, two indices $i$ and $j, i$ is to the left of $j$ if and only if $i<j . i$ is below $j$ if and only if $s y(i) \rho s y(j)$.

In this section $*$ and $\rho$ have the same meanings as in Definition 2. OSY is the sorted list of the elements in $S Y . S D(i)$ is the general prefix product of all the elements in $S F$, whose indices are to the left of $i$ and below $i, 1 \leq i \leq k$. In other words, $S D(i)=s f\left(j_{1}\right) *$ $s f\left(j_{2}\right) * \cdots s f\left(\bar{j}_{t}\right), 1 \leq i \leq k$ where, $\left\{j_{1}, j_{2}, \cdots, j_{t}\right\}$ is the set of all indices $j$ such that $j<i$ and $s y(j) \rho s y(i)$ and $j_{r}<j_{r+1}, 1 \leq r<t$. Similarly, $S P(i)=s f\left(j_{1}\right) *$ $s f\left(j_{2}\right) * \cdots s f\left(j_{t}\right), 1 \leq i \leq k$ where, $\left\{j_{1}, j_{2}, \cdots, j_{q}\right\}$ is the set of all indices $j$ such that $s y(j) \rho s y(i)$ (indices below $i)$ and $S E(i)=s f\left(j_{1}\right) * s f\left(j_{2}\right) * \cdots s f\left(j_{t}\right), 1 \leq$ $i \leq k$ where, $\left\{j_{1}, j_{2}, \cdots, j_{q}\right\}$ is the set of all indices $j$ such that $(s y(j) \rho s y(i))$ or $(s y(j)=s y(i))$ and $j_{r}<j_{r+1}, 1 \leq r<t$ (indices below or equal to $i$ ). We define the following function:

Function $S E Q G P C(S F, S Y, k):(O S Y, S P, S D, S E)$ The function takes as input two arrays $S F$ and $S Y$ of length $k$ and outputs four arrays, $O S Y, S P, S D$ and $S E$, each of length $k$.

At every stage of the recursion, each of the input sequences (refer to Definition 2) $S F=(s f(1), s f(2), \ldots$, $s f(k))$ and $S Y=(s y(1), s y(2), \ldots, s y(k))$ are divided into two halves. Let $L S F=\left(s f(1), s f(2), \ldots, s f\left(\frac{k}{2}\right)\right)$ and $R S F=\left(s f\left(\frac{k}{2}+1\right), \ldots, s f(k)\right)$. Similarly, let $L S Y=\left(s y(1), s y(2), \ldots, s y\left(\frac{k}{2}\right)\right)$ and $R S Y=\left(s y\left(\frac{k}{2}+\right.\right.$ $1), \ldots, s y(k))$. The two subproblems, namely, the left subproblem with input sequences $L S F$ and $L S Y$, and the right subproblem with input sequences $R S F$ and $R S Y$ are solved recursively using function $S E Q G P C$. The solution for the left subproblem are four arrays each of length $\frac{k}{2}$, denoted by $L O S Y, L S D, L S P, L S E$ in order. Similarly, the solution for the right subproblem are four arrays each of length $\frac{k}{2}$, denoted by $R O S Y, R S D, R S P, R S E$ in order.

The next step is to merge these two solutions to form the four arrays $O S Y, S D, S P, S E$ for the problem with input sequences $S F$ and $S Y$. Clearly $O S Y$ can be obtained by merging the two sorted lists LOSY and $R O S Y$. For every element $s y(i)$ in $L S Y$, i.e., $1 \leq i \leq \frac{k}{2}$, we find the indices $\operatorname{LPOS}(i)$ and $R P O S(i)$ of those two elements in $R S Y$, such that $s y(i)$ is in between $s y(\operatorname{LPOS}(i))$ and $s y(R P O S(i))$ in $O S Y$ and no other element of $R S Y$ is in between $s y(L P O S(i))$ and $s y(R P O S(i))$ in $O S Y$. Similarly, for every element $s y(i)$ in $R S Y$, i.e., $\frac{k}{2}+1 \leq i \leq k$, we find the 
indices $L P O S(i)$ and $R P O S(i)$ of those two elements in $L S Y$, such that $s y(i)$ is in between $s y(L P O S(i))$ and $s y(R P O S(i))$ in $O S Y$ and no other element of $L S Y$ is in between $s y(\operatorname{LPOS}(i))$ and $s y(R P O S(i))$ in $O S Y$. It is straightforward to see that, $\operatorname{LPOS}(i)=$ rank of $s y(i)$ in $O S Y$ - rank of $s y(i)$ in LOSY, for $s y(i) \in L S Y$, i.e., $1 \leq i \leq \frac{k}{2}$. Similarly, $\operatorname{LPOS}(i)$ $=$ rank of $s y(i)$ in $O S Y$ - rank of $s y(i)$ in $R O S Y$, for $s y(i) \in R S Y$, i.e., $\frac{k}{2}+1 \leq i \leq k$. RPOS $(i)=$ $\operatorname{LPOS}(i)+1,1 \leq i \leq k$. Now we present the following lemma.

\section{Lemma 1 (Merge Lemma [6])}

1. If $1 \leq i \leq \frac{k}{2}$, then,

a. $S D(i)=L S D(i)$

b. $S P(i)=L S P(i) * R S P(L P O S(i))$,

if $s y(\operatorname{LPOS}(i))=s y(i)$

c. $S P(i)=L S P(i) * R S E(L P O S(i))$, if $s y(L P O S(i)) \rho s y(i)$

d. $S E(i)=L S E(i) * R S E(L P O S(i))$, if $s y(L P O S(i))=s y(i)$

e. $S E(i)=L S E(i) * R S P(R P O S(i))$, if $(s y(L P O S(i)) \rho s y(i)) \wedge(s y(i) \rho s y(R P O S(i)))$

f. $S E(i)=L S E(i) * R S E(R P O S(i))$, if $(s y(\operatorname{LPOS}(i)) \rho s y(i)) \wedge(s y(i)=s y(R P O S(i)))$

2. If $\frac{k}{2}+1 \leq i \leq k$, then,

a. $S D(i)=\operatorname{LSP}(\operatorname{LPOS}(i)) * R S D(i)$, if $s y(L P O S(i))=s y(i)$

b. $S D(i)=L S E(L P O S(i)) * R S D(i)$, if $s y(L P O S(i)) \rho s y(i)$

c. $S P(i)=L S P(L P O S(i)) * R S P(i)$, if $s y(L P O S(i))=s y(i)$

d. $S P(i)=L S E(\operatorname{LPOS}(i)) * R S P(i)$, if $s y(L P O S(i)) \rho s y(i)$

e. $S E(i)=L S E(\operatorname{LPOS}(i)) * R S E(i)$, if $s y(L P O S(i))=s y(i)$

f. $S E(i)=L S P(R P O S(i)) * R S E(i)$, if $(s y(L P O S(i)) \rho s y(i)) \wedge(s y(i) \rho s y(R P O S(i)))$

g. $S E(i)=L S E(R P O S(i)) * R S E(i)$, if $(s y(L P O S(i)) \rho s y(i)) \wedge(s y(i)=s y(R P O S(i)))$

Proof: We will prove Case 2a. For a given index $i$ such that $\frac{k}{2}+1 \leq i \leq k$, let $\left\{j_{1}, j_{2}, \cdots, j_{t}\right\}$ is the set of all indices $j$ such that $1 \leq j \leq \frac{k}{2}$ and $s y(j) \rho s y(i)$ and $j_{r}<j_{r+1}, 1 \leq r<t$. If $s y(L P O S(i))=s y(i)$, then, it is straightforward to see that $\operatorname{LSP}(\operatorname{LPOS}(i))=$ $f\left(j_{1}\right) * f\left(j_{2}\right) * \cdots * f\left(j_{t}\right)$. This and definition of $R S D(i)$ imply $S D(i)=L S P(L P O S(i)) * R S D(i)$, if $s y(\operatorname{LPOS}(i))=s y(i)$. The proof for the other cases is similar.

From the above discussion and Lemma 1 we see that the merge step can be implemented in $O(k)$ time. Hence the whole algorithm takes $O(k \log k)$ time.

Theorem 3 Function SEQGPC $(S F, S Y, k)$ takes $O(k \log k)$ time.

\subsection{The Main Algorithm}

Let us assume the two input sequences $F=(f(1)$, $f(2), \ldots, f(t(n / p)))$ and $Y=(y(1), y(2), \ldots, y(t(n / p))))$ be $I E D$ stored on the arrays $S F$ and $S Y$ respectively in the processors $P R_{i}, P R_{i+1}, \ldots, P R_{i+t-1}$. We also assume that the sequences $O S Y, S P, S D, S E$, $L P O S, R P O S$ (as defined earlier are IED stored on the arrays $S O S Y, S S P, S S D$,SSE, SLPOS, $S R P O S$ respectively. The following recursive function solves the $G P C$ problem for above input sequences $S F$ and $S Y$.

Function Block $G P C(S F, S Y, i, t):(D A T A)$

Input: Two input sequences $F=\left(s f(1), \ldots, s f\left(t\left\lceil\frac{n}{p}\right\rceil\right)\right.$ and $Y=\left(s y(1), \ldots, s y\left(t\left\lceil\frac{n}{p}\right\rceil\right)\right.$, each $I E D$ stored on the arrays $S F$ and $S Y$ respectively (i.e., stored in the processors $P R_{i}, P R_{i+1}, \ldots, P R_{i+(t-1)}$ ).

Output: $S O S Y$ is the sorted list of the elements in $S Y$. Suppose $S O S Y[j, k]=S Y[l, q]$, then, DAT $A[j, k]$ stores the ordered list $\langle S Y[l, q], D A T[l, q]\rangle$, where $D A T[l, q]=<q, l, S S F[l, q], S S D[l, q], S S P[l, q], S S E[l, q]>$ $D A T A$ is IED stored in the processors $P R_{i}, P R_{i+1}, \ldots, P R_{i+(t-1)}$.

Begin /* W.l.g we assume $t$ is a power of two $* /$

1. If $(t=1)$ then $/ *$ There is only one block $* /$

Processor $P R_{i}$ will do /* Solved sequentially */ $(S O S Y, S S P, S S D, S S E)=S E Q G P C\left(S F, S Y, M_{a x}\right)$.

For $j=1$ to $\operatorname{Max}_{i}$ do If $S O S Y[j, i]=S Y[k, i]$ then

$D A T A[j, i]=<S Y[k, i],<i, k, S S F[k, i], S S D[k, i]$, $S S P[k, i], S S E[k, i]>>$

return $(D A T A)$; exit.

2. Do Steps 2.1 and 2.2 in parallel $/ * t>1 * /$

/* recursively solving left and right halves $*$ /

$2.1(L D A T A)=\operatorname{Block} G P C\left(S F, S Y, i, \frac{t}{2}\right)$

$2.2(R D A T A)=B l o c k G P C\left(S F, S Y, i+\frac{t}{2}, \frac{t}{2}\right)$

3. $(B P L, T F)=R A N K P(L D A T A, R D A T A, i, t)$

/* From the definition of function RANKP we see that $B P L[j, i]$ is a two tuple and the sequence consisting of the first entries of all the tuples is the sorted list SOSY. *I 4. If $S O S Y[j, k]=S Y[l, q]$, then $B P L[j, k]=\langle S Y[l, q]$, dat $\rangle$ From the function RANKP and definitions of $L D A T A$ and $R D A T A$ we see that $T F[j, k]$ contains the necessary data as suggested in Lemma 1 for calculating $S S F[l, q]$, $S S D[l, q], S S P[l, q], S S E[l, q]$. Hence we compute $D A T A[j, k]=<S Y[l, q],<q, l, S S F[l, q], S S D[l, q]$, 5. $\operatorname{return}(D A T A)$ $S S P[l, q], S S E[l, q]>$.

End.

Let $T_{\text {comp }}(t)$ be the computation time taken by Block $G P C(S F, S Y, i, t)$. From Theorem $3 T_{\text {comp }}(1)=$ $O\left(\frac{n}{p} \log \left(\frac{n}{p}\right)\right)$ (Step 1 of the function). Step 4 takes $O\left(\frac{n}{p}\right)$ computation time. From Theorem 2 we see that, Step 4 takes $O\left(\frac{n \log _{2} t}{p}+\frac{\tau \log _{2} t}{\sigma m \log _{2}\left(\frac{\tau}{\sigma m}+1\right)}\right)$ time. Hence, $T_{\text {comp }}(t)=T_{\text {comp }}\left(\frac{t}{2}\right)+O\left(\frac{n \log _{2} t}{p}+\frac{\tau \log _{2} t}{\sigma m \log _{2}\left(\frac{\tau}{\sigma m}+1\right)}\right)=$ $O\left(\frac{n}{p}\left(\log \left(\frac{n}{p}\right)+\log ^{2} t\right)+\frac{r \log _{2}^{2} t}{\sigma m \log _{2}\left(\frac{\tau}{\sigma m+1)}\right)}\right.$. Similarly, 
let $T_{\text {comm }}(t)$ be the communication time taken by BlockGPCMerge $(S F, S Y, i, t) . \quad T_{\text {comm }}(1)=0 . \mathrm{We}$ can easily see that $T_{\text {comm }}(t)=T_{\text {comm }}\left(\frac{t}{2}\right)+O((\tau+$ $\left.\left.\sigma m\left\lceil\frac{n}{p m}\right\rceil\right) \log t\right)=O\left(\left(\tau+\sigma m\left\lceil\frac{n}{p m}\right\rceil\right) \log ^{2} t\right)$. Hence, the following theorem.

Theorem 4 Function BlockGPC(SF,SY,i,t) takes $O\left(\frac{n}{p}\left(\log \left(\frac{n}{p}\right)+\log ^{2} t\right)+\frac{\tau \log _{2}^{2} t}{\sigma m \log _{2}\left(\frac{r}{\sigma m}+1\right)}\right)$ computation time and $O\left(\left(\tau+\sigma m\left\lceil\frac{n}{p m}\right\rceil\right) \log ^{2} t\right)$ communication time.

Function $B D S M G P C(F, Y):(S D)$

Input: Two input sequences $f=(f(1), \ldots, f(n)$ and $y=(y(1), \ldots, y(n)$, ach $I E D$ stored on the arrays $F$ and $Y$ respectively.

Output: The sequence $d=(d(1), d(2), \ldots, d(n))$ the general prefixes as defined in Definition 2, IED stored on the array $D$.

Begin

1. DATA $=$ BlockGPC $(F, Y, 0, p)$

2. For every processor $P R_{i}, 0 \leq i \leq p-1$ do in Parallel For $1 \leq j \leq n / p$ d $\phi$

Let $\left\langle a_{1},\left\langle a_{2}, a_{3}, b_{1}, b_{2}, b_{3}, b_{4}\right\rangle\right\rangle=D A T A[j, i]$

$/ * a_{1}=S Y[u, v], a_{2}=v$ and $a_{3}=u$ and $b_{2}=D[u, v] * /$

Let $A[j, i]=\left\langle a_{2}, a_{3}, b_{2}\right\rangle$

3. $\left(A^{\prime}, c\right)=R A N D O M R O U T E(A)$.

$/ *$ In the above call $\phi=1$ as exactly $\frac{n}{p}$ data are

destined for any processor (refer to Theorem A.1) */

4. For every processor $P R_{i}, 0 \leq i \leq p-1$ do in Parallel

For $1 \leq j \leq c \operatorname{Max}$, do

Let $<a_{1}, a_{2}, a_{3} \ngtr=A^{\prime}[j, i]$

End.

$D\left(a_{2}, a_{1}\right)=a_{3}$

The above function and Theorems A.1 and 4 imply the following theorem.

Theorem 5 Function $B D S M G P C(F, Y)$

takes $O\left(\frac{n}{p}\left(\log \left(\frac{n}{p}\right)+\log ^{2} p\right)+\frac{\tau \log _{2}^{2} p}{\sigma m \log _{2}\left(\frac{\tau}{\sigma m}+1\right)}\right)$ expected computation time and $O\left(\left(\tau+\sigma m\left\lceil\frac{n}{p m}\right\rceil\right) \log ^{2} p\right)$ expected communication time.

\section{Applications}

\subsection{Sorting}

The following transformation is given in [6]. Let $x(1), x(2), \ldots, x(n)$ be the $n$ numbers to be sorted. Set $y(i)=x(i), f(i)=1, \rho=\leq$ and $*=+$ (summation) in Definition 2 and we perform the $G P C$ to compute $D_{i}, 1 \leq i \leq n$. Clearly the prefix $D_{i}$ gives the number of elements $j$ such that $x(j) \leq x(i)$ and $j<i$. Again set $y(i)=x(n-i), f(i)=1, \rho=<$ and $*=+$ (summation) and we perform the GPC to compute $D_{i}^{\prime}, 1 \leq i \leq n$. Glearly the prefix $D_{n-i}^{\prime}$ gives the number of elements $j$ such that $x(j)<x(i)$ and $j>i$. Now $D_{i}+D_{n-i}^{\prime}+1$ gives the position /of $x_{i}$ in the sorted list. We now use the function Randomroute to route the $x_{i}$ 's such that the sorted list is IED stored on the array, say $S$, on a $p$-processor $B D M$. This and Theorem 5 imply part of Theorem 6 .

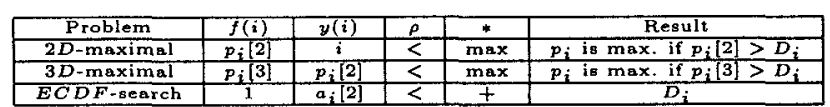

Figure 2: Mapping of Dominance and Searching Problems onto $G P C$.

\subsection{Computational Geometry}

Given a set $S=\left\{p_{1}, p_{2}, \ldots, p_{n}\right\}$ of $n$ points in $D$ dimensional space. A point $p_{i} D$-dominates a point $p_{j}$ if and only if $p_{i}[k]>p_{j}[k]$, for all $1 \leq k \leq D$, where $p[k]$ denotes the $k^{t h}$ coordinate of a point $p$. The 2-dimensional ECDF searching problem consists of computing for each $p$ in a 2-dimensional point set $S$, the number of points of $S 2$-dominated by $p$. The $D$-dimensional maximal elements problem is to determine the points in a given $D$-dimensional point set $S$, which are $D$-dominated by no other point. For each of the problems we sort the points in $S$ by first coordinate and denote the lists sorted in ascending and descending order by $a_{1}, a_{2}, \ldots, a_{n}$ and $b_{1}, b_{2}, \ldots, b_{n}$ respectively. Figure 2 shows the transformation for the above mentioned problems [6].

Given two point sets $A$ and $B$, the 2-set dominance problem in two dimensions is the problem of counting for each point $p$ from $B$ the number of points from $A$ that $p$ 2-dominates. Let $|A|=n$ and $|B|=m^{\prime}$. Let $a_{1}, a_{2}, \ldots, a_{m^{\prime}+n}$ be the list of the $m^{\prime}+n$ points belonging to the set $A \cup B$, sorted in ascending order of their first coordinate. If $a_{i} \in A$, then, $f(i)=1$ else $f(i)=0 . y(i)=a_{i}[2], \rho=<$ and $*=+$. The result is $D_{i}$, i.e., point $a_{i} \in B$ 2-dominates $D_{i}$ number of points in $A[6]$. Hence the following theorem.

Theorem 6 Sorting problem, ECDF Searching problem, $2 D$ and $3 D$ maximal elements problem and 2set Dominance Counting problem can be solved in $O\left(\frac{n}{p}\left(\log \left(\frac{n}{p}\right)+\log ^{2} p\right)\right)$ expected computation time and $O\left(\left(\tau+\sigma m\left\lceil\frac{n}{p m}\right\rceil\right) \log ^{2} p\right)$ expected communication time on a p-processor DSM system using the BDM model.

\subsection{Graph Theory}

Permutation Graphs: Given a permutation $\pi$ of the integer set $\mathrm{V}=\{1,2,3, \ldots, n\}, \pi$ defines a graph $G_{p}=$ $(\mathrm{V}, \mathrm{E})$, where $(i, j) \in \mathrm{E}$ if and only if $(i-j) *\left(\pi^{-1}(i)-\right.$ $\left.\pi^{-1}(j)\right)<0$, where $\pi^{-1}(k)$ denotes the position of $k$ in $\pi$. A graph $\mathrm{G}$ is a permutation graph if and only if it is isomorphic to some $G_{\pi}$. We assume that the permutation $\pi$ is $I E D$ stored in the array $P$ and the inverse permutation $\pi^{-1}$ is $I E D$ stored in the array $P I$. Given $P$ it is straightforward to compute $P I$ on a $D S M$ system using the function RandomRoute. The arrays $L S V, M S V, C N U M, W$ and $V$ defined in [1] can be computed using the function BDPRECOMP. This, Theorem A.2 and the algorithms CONCOMP, Cutvertices, Bridges presented in [1] imply a part of Theorem 7 .

Binary Tree Reconstruction: We assume that the preorder traversal pre and inorder traversal in are 
$I E D$ stored in the array $P R E$ and $I N$ respectively. It is straightforward to implement the algorithm presented in [5] using the functions BDPRECOMP and RandomRoute to reconstruct the binary tree represented by these traversals. Hence the following Theorem.

Theorem 7 The problems of finding Connected Components, Cut Vertices and Bridges of a given Permutation graph of $n$ vertices and the problem of reconstructing a binary tree from its Inorder and Preorder traversals can be solved in $4 \tau\left\lceil\frac{\log _{2} p}{\log _{2}\left(\frac{\tau}{\sigma m}+1\right)}\right\rceil+\tau+\sigma m+O\left(\frac{n}{p}\right)$ expected communication time and $O\left(\frac{n}{p}+\frac{\tau \log _{2} p}{\sigma m \log _{2}\left(\frac{\tau}{\sigma m}+1\right)}\right)$ expected computation time on a p-processor DSM system using the $B D M$ model.

\section{Conclusion}

In this paper we have presented an efficient algorithm for $G P C$ on $D S M$ systems using the $B D M$ model with applications. Since sorting is transformed into an instance of $G P C, G P C$ has a lower bound of $\Omega(n \log n)$ at least for some specific choice of operators $*$. As seen before, if $n>p \log p$, our algorithm for $G P C$ takes $O\left(\frac{n \log n}{p}\right)$ computation time. Hence, we have achieved optimal speed up in computation for the sorting problem for such realistically larger values of $n$. Further, our method is conceptually simpler than the algorithms presented in [3] for sorting. From the analytical expressions for the time taken by $B D P R E C O M P$ and BlockMerge, we see that the time taken will increase with the number of processors $(p)$ for smaller size of the inputs. From Corollary 1 we see that the computation time of BlockMerge is $O\left(n \log _{2} p / p\right)$ and hence we cannot expect any scalability from $p=2$ to $p=4$. It is interesting to note that the timing measurements agree with the theoretical predictions and the graphical plots illustrates the scalability (refer Figure 3). It is worth noting that the merge function is recursive and it executes on a DMS which might serve as a pointer for incorporating parallel recursion in automatic parallelising compilers.

\section{Appendix A}

Function RANDOMROUTE $(A):\left(A^{\prime}, c\right)$

Input: $A\left[1:\left\lceil\frac{n}{p}\right\rceil, 0: p-1\right]$ is the input array $I E D$ stored on a $p$-processor $B D M$ machine, such that each element of $A$ consists of a packet $\left(i\right.$, data $\left._{i}\right)$ of constant size, where $i$ is the index of the processor to which data $_{i}$ has to be routed. $\alpha$ is a constant such that no processor is the destination of more than $\alpha\left\lceil\frac{n}{p}\right\rceil$ elements on the whole.

Output: $A^{\prime}\left[1: c\left\lceil\frac{n}{p}\right\rceil, 0: p-1\right]$ is the output array holding the routed data, IED stored on a $p$ processor $B D M$ machine, such that all the data with the processor $P R_{i}, 0 \leq i \leq p-1$, as the destination will be available in one of the locations $A^{\prime}[j, i]$, $1 \leq j \leq c\left\lceil\frac{n}{p}\right\rceil$ in the processor $P R_{i}$, where $c$ is larger than $\max \left\{1+\frac{1}{\sqrt{2}}, \alpha+\frac{\sqrt{\alpha}}{2}\right\}$. The function stores a copy of $c$ in every processor $P R_{i}, 0 \leq i \leq p-1$.

The function is implemented using the Randomized_Routing algorithm suggested in [3].

Theorem A.1 Function RANDOMROUTE $(A)$ completes within $2\left(\tau+c\left\lceil\frac{n}{p}\right\rceil\right)$ communication time and $O\left(c\left\lceil\frac{n}{p}\right\rceil\right)$ computation time with high probability, where $c$ is larger than $\max \left\{1+\frac{1}{\sqrt{2}}, \alpha+\frac{\sqrt{\alpha}}{2}\right\}, p^{2}<\frac{n}{6 \ln n} \square$

Function $\operatorname{BDPRECOMP}(A, r, \nabla):\left(A^{\prime}\right)$.

Input: Given a sequence of ordered pairs $<a_{1}$, data $_{1}>,<a_{2}$, data $_{2}>, \cdots,<a_{t(n / p)}, \operatorname{data}_{t(n / p)}>$, $I E D$ stored on the array $A$, on $t$ processors of a $B D M$ machine, data $a_{i}$ is the data (if any) associated with $a_{i}$, $1 \leq i \leq t(n / p) . \nabla$ is a binary associative operator $\epsilon$ $\{+, \operatorname{Min}, \operatorname{Max}, \ldots\}$.

Output: A sequence of ordered pairs $<a_{1}^{\prime}$, dat $\left.a_{1}^{\prime}\right\rangle$, $<$ $a_{2}^{\prime}$, data $a_{2}^{\prime}>, \cdots,<a_{t(n / p)}^{\prime}$, dat $a_{t(n / p)}^{\prime}>, I E D$ stored on the array $A^{\prime}$, on a $p$-processor $B D M$ machine, where, $a_{i}^{\prime}=\nabla_{k=1}^{i} a_{k}$ and dat $a_{i}^{\prime}$ is the data associated with $a_{i}^{\prime}$ (if any).

From Theorem 9 of [4] we infer the following theorem.

Theorem A.2 ([4]) Given a sequence $\left(a_{1}, a_{2}, \ldots\right.$, $\left.a_{t(n / p)}\right)$ of numbers IED stored on $t$ processors of $a$ $B D M$ machine, we can compute the prefix sums ps $s_{i}=$ $\sum_{j=1}^{i} a_{j}, 1 \leq i \leq r$, in $4 \tau\left\lceil\frac{\log _{2} t}{\log _{2}\left(\frac{\tau}{\sigma m}+1\right)}\right\rceil+\tau+\sigma m$ communication time and $O\left(\frac{n}{p}+\frac{\tau \log _{2} t}{\sigma m \log _{2}\left(\frac{\tau}{\sigma m}+1\right)}\right)$ computation time. This complexity holds for prefix maxima, prefix minima and similar associative operators.

\section{References}

[1] K. Arvind, V. Kamakoti, and C. Pandurangan. Efficient parallel algorithms for permutation graphs. Journal of Parallel and Distributed Computing, 26:116-124, 1995.

[2] K. E. Batcher. Sorting networks and their applications. In Proceedings AFIPS 32nd Spring Joint Computer Conference, pages 307-314, 1968.

[3] F. J. Jaja and K. W. Ryu. The block distributed memory model. IEEE Transactions on Parallel and Distributed Systems, 7(8):830-840, 1996.

[4] V. Kamakoti and N. Balakrishnan. Efficient randomized algorithm for the closest pair problem on distributed shared memory systems. Report, SERC, IISc, Bangalore - 560 012, India, 1996.

[5] V. Kamakoti and C. Pandurangan. An optimal algorithm for reconstructing a binary tree. Information Processing Letters, 42(2):113-115, 1992.

[6] F. Springsteel and I. Stojmenovic. Parallel general prefix computations with geometric, algebraic and other applications. Report, University of Ottawa, Computer Science, Ottawa, Ontario K1N 9B4, Canada. 

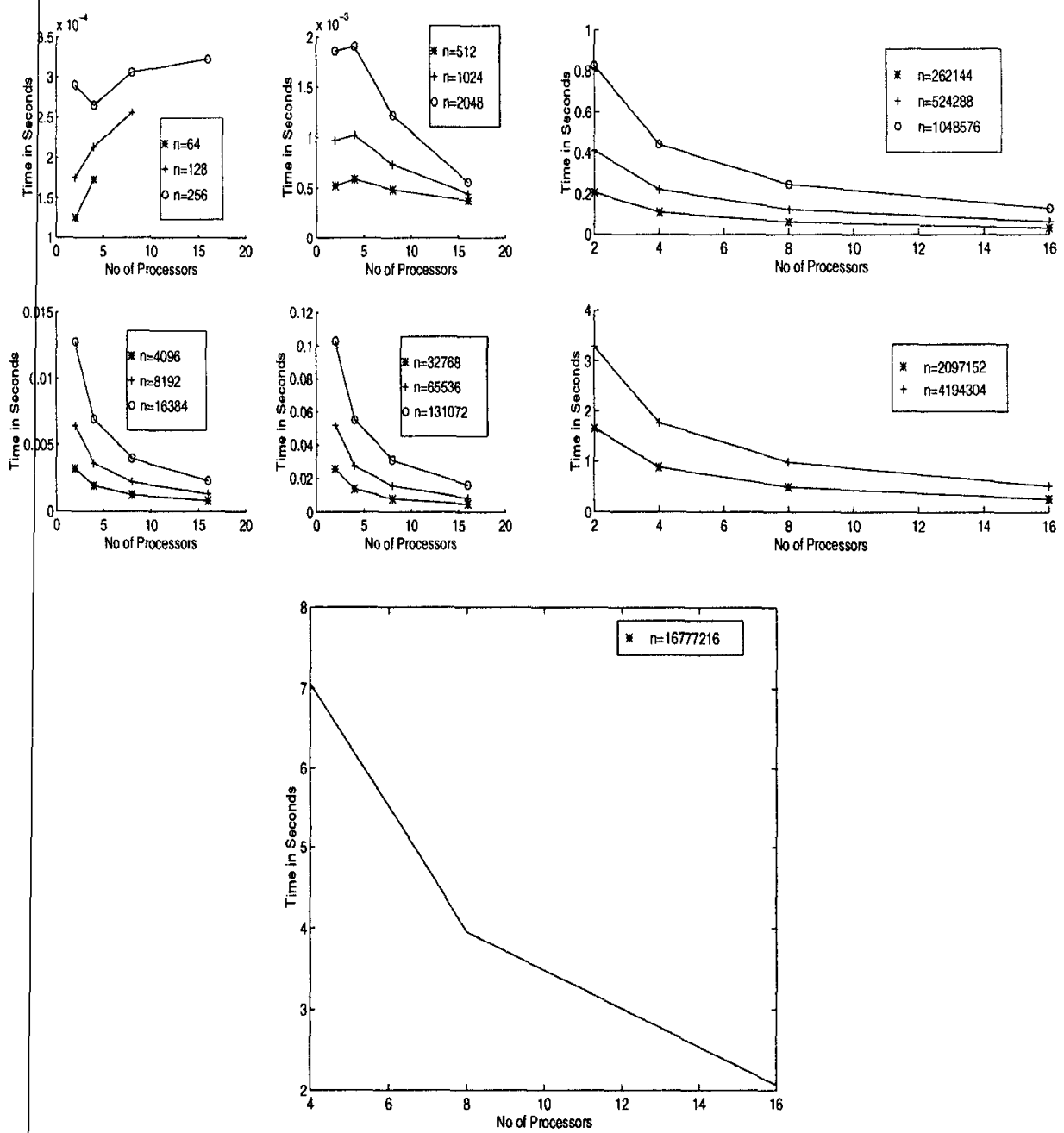

(a). Prefix Sum on IBM SP2
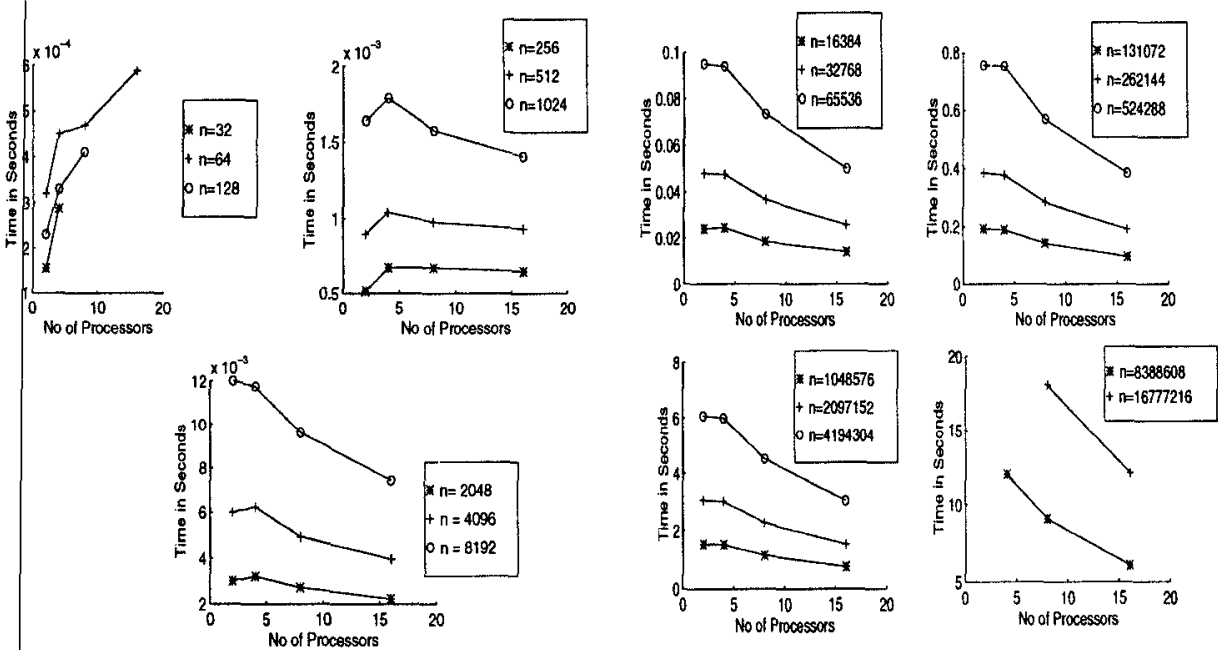

Figure 3: (b). Block Merging on IBM SP2 